\title{
CHAINS, NULL-CHAINS, AND CR GEOMETRY
}

\author{
LISA K. KOCH
}

\begin{abstract}
A system of distinguished curves distinct from chains is defined on indefinite nondegenerate CR hypersurfaces; the new curves are called nullchains. The properties of these curves are explored, and it is shown that two sufficiently nearby points of any nondegenerate CR hypersurface can be connected by either a chain or a null-chain.
\end{abstract}

\section{INTRODUCTION}

Two real hypersurfaces in $\mathbb{C}^{n+1}$ are, in general, inequivalent under biholomorphic transformations on the ambient $\mathbb{C}^{n+1}$ if $n \geq 1$. E. Cartan (for $n=1$ ) and Chern-Moser (for $n \geq 1$ ) have defined complete systems of invariants for such CR hypersurfaces; the equality of the invariants is necessary and sufficient for the equivalence of the hypersurfaces $[\mathrm{C}, \mathrm{CM}])$. Among these invariants is a system of curves called chains. Through any point of a nondegenerate CR hypersurface, in any direction transverse to its holomorphic tangent space at that point, there passes a unique chain.

The study of invariants on CR hypersurfaces is CR geometry, and it has many tempting parallels with Riemannian geometry [CM]. In particular, it is natural to compare chains to geodesics, and it was asked in [BS] whether a point of a CR hypersurface can be connected to any point of some neighborhood by a chain. It is now known that chains do connect nearby points of strictly pseudoconvex CR hypersurfaces [J, Kr, Ko]). But, as Jacobowitz has observed [J], this result cannot hold for a nondegenerate CR hypersurface of general signature.

In this paper, it will be shown that the system of chains on indefinite nondegenerate CR hypersurfaces is, in a way, incomplete. We will define new CR invariant curves called null-chains which complete the system on these hypersurfaces. The curves of the completed system will be shown to connect pairs of nearby points of any nondegenerate CR manifold regardless of signature.

The author would like to thank Jürgen Moser and C. Denson Hill for a helpful conversation.

\section{CR MANIFOLDS AND CHAINS}

Let $M$ be an odd-dimensional orientable smooth manifold of dimension $2 n+1$. An almost $C R$ structure on $M$ is an $n$-dimensional complex subbundle

Received by the editors October 4, 1988 and, in revised form, April 8, 1991. 1991 Mathematics Subject Classification. Primary 32F25; Secondary 53C50.

Research supported in part by NSF grant \#DMS-8703275. 
$T_{1,0}$ of the complexified tangent bundle $\mathbb{C} T M$ of $M$ such that $T_{1,0} \cap T_{0,1}=$ $\{0\}$, where $T_{0,1}=\overline{T_{1,0}}$. We set $\mathscr{H}=\Re\left(T_{1,0} \oplus T_{0,1}\right) ; \mathscr{H}$ is a real $2 n$ dimensional subbundle of $T M$, and we call $\mathscr{H}_{x}$ the holomorphic tangent space of $M$ at $x$. It carries a natural complex structure $\mathscr{J}$ given by $\mathscr{J}(V+\bar{V})=$ $i(V-\bar{V})$ for $V \in T_{1,0}$; this $\mathcal{J}$ is called a $C R$ structure tensor on $M$. The almost CR structure is said to be a $C R$ sturcture if it is formally integrable in the sense that $\left[T_{1,0}, T_{1,0}\right] \subset T_{1,0} . M$ together with such a CR structure is said to be an abstract $C R$ manifold (of hypersurface type).

Without referring to the complexified tangent space, we may equivalently define an almost CR structure on $M$ as a field of tangent hyperplanes $\mathscr{H}$ on $M$ together with a smooth map $\mathscr{J}: \mathscr{H} \rightarrow \mathscr{H}$ which is linear on each $\mathscr{H}_{x}$, $x \in M$, and such that $\mathscr{J}^{2}=-\left.\mathrm{id}\right|_{\mathscr{H}}$. The integrability of the structure is equivalent to the vanishing of the Nijenhuis torsion tensor

$$
N(X, Y)=[\mathscr{J} X, \mathscr{J} Y]-[X, Y]-\mathscr{J}([X, \mathscr{J} Y]+[\mathscr{J} X, Y])
$$

for $X, Y \in \mathscr{H}$.

Let $\theta \in T^{*} M$ be a one-form which annihilates $\mathscr{H}$. A (an almost) CR structure, together with such a one-form, is called a (an almost) pseudo-Hermitian structure on $M[\mathrm{~W}]$. The $\mathrm{CR}$ (almost $\mathrm{CR}$ ) structure is said to be nondegenerate if $\theta \wedge(d \theta)^{n} \neq 0$; this condition is independent of the choice of $\theta$. For the rest of this paper, the words "CR manifold" will mean "nondegenerate $C R$ manifold of hypersurface type".

With respect to a choice of pseudo-Hermitian one-form $\theta$ on $M$, the Levi form $\mathrm{L}_{\theta}$ of $M$ is the Hermitian form on $T_{1,0}$ given by

$$
\mathrm{L}_{\theta}(V, W)=i d \theta(V, \bar{W}) .
$$

This form $\mathrm{L}_{\theta}$ depends on the choice of $\theta$, but it changes only by a conformal multiple if $\theta$ is changed; thus, the signature of $\mathrm{L}_{\theta}$ is invariant. If $\mathrm{L}_{\theta}$ is (positive) definite, $M$ is said to be strictly pseudoconvex. The Levi form $\mathrm{L}_{\theta}$ as defined above gives rise to a real symmetric form on $\mathscr{H}$, which we also call $\mathrm{L}_{\theta}$.

In 1907, Poincare [P] observed that real hypersurfaces in $\mathbb{C}^{n}$ may be locally inequivalent under biholomorphic transformations if $n \geq 2$. He conjectured that certain invariants could be associated to such hypersurfaces; the signature of the Levi form is one such invariant. Cartan [C] found a system of invariants for hypersurfaces in $\mathbb{C}^{2}$ whose equality is necessary and sufficient for the equivalence of the hypersurfaces.

Cartan also defined a biholomorphically invariant system of curves on nondegenerate hypersurfaces in $\mathbb{C}^{2}$, called chains. On the hyperquadric $Q^{3} \subset \mathbb{C}^{2}$, given by

$$
Q^{3}=\left\{\left(z_{0}, z_{1}\right) \in \mathbb{C}^{2}: \Im z_{0}=\left|z_{1}\right|^{2}\right\},
$$

the chains are the intersections of the hypersurface with affine complex lines. In general, chains are the solutions of a system of differential equations [J]. To give this system explicitly for a three-dimensional CR manifold $M$, choose a pseudo-Hermitian structure $\theta$ on $M$, and let us define $\theta^{1}$ to be the complex one-form which annihilates all vectors of the form $X+i \mathcal{J} X$, for $X \in \mathscr{H}$. For any complex-valued function $c$ on $M$, define $c_{0}, c_{1}$, and $c_{\overline{1}}$ by $d c=$ 
$c_{0} \theta+c_{1} \theta^{1}+c_{\overline{1}} \overline{\theta^{1}}$. Then the chains on $M$ are the curves on $M$ which solve the equations

(1) $\theta^{1}=-\mu \theta$,

(2) $d \mu=\left(i \mu|\mu|^{2}+\frac{1}{2} i c \mu-\frac{1}{6} \bar{l}-\bar{b}|\mu|^{2}\right) \theta$,

where $b$ satisfies $d \theta=i \theta^{1} \wedge \overline{\theta^{1}}+b \theta \wedge \theta^{1}+\bar{b} \theta \wedge \overline{\theta^{1}}$, and $c$ and $l$ are given by $c=\overline{b_{1}}$ and $l=c_{1}-b c-2 i b_{0}$. Through any point of a hypersurface in $\mathbb{C}^{2}$, in any direction transverse to the holomorphic tangent space $\mathscr{H}$, there passes a unique chain. Chains carry a distinguished parameter which is defined up to a real projective transformation.

The work of Cartan was extended by Chern and Moser [CM] to all nondegenerate abstract CR manifolds of hypersurface type. Through any point of such a CR manifold, in any direction transverse to the holomorphic tangent plane $\mathscr{H}$, there passes a unique chain. Chains carry a distinguished parameter defined up to a real-projective transformation.

The Moser normal form for the defining equation of a CR hypersurface $M$ is produced by osculating $M$ with a biholomorphic image of the hyperquadric of appropriate dimension and signature. This osculation takes place at a point $x \in M$ along a chain passing through $x$. Cheng [Ch] has recently shown that any chain-preserving diffeomorphism of a nondegenerate CR manifold is either a $\mathrm{CR}$ or an anti-CR isomorphism.

The hyperquadric $Q_{p, q} \subset \mathbb{C}^{n+1}$ of Levi signature $p, q$ (where $p+q=n$ ) is

$$
Q_{p, q}=\left\{\left(z_{0}, \ldots, z_{n}\right): \Im z_{0}=\left|z_{1}\right|^{2}+\cdots+\left|z_{p}\right|^{2}-\left|z_{p+1}\right|^{2}-\cdots-\left|z_{p+q}\right|^{2}\right\} .
$$

The chains on $Q_{p, q}$ are its intersections with affine complex lines in $\mathbb{C}^{n+1}$, when the intersection defines a curve. (It may happen, if $p, q \geq 1$, than the complex line is a subset of $Q_{p, q}$, and in this case the intersection does not define a chain.)

\section{The FefFERMAN CORRESPONDENCE}

Another very useful way of describing chains is provided by the Fefferman correspondence [F, BDS]. To any nondegenerate CR manifold $M$ of Levi signature $p, q$ there corresponds a conformal pseudo-Riemannian manifold $F$, of signature $2 p+1,2 q+1$, whose underlying space is a certain circle bundle over $M$. (The conformal factors are restricted to be functions on $M$, that is, constant on the fibers.) The conformal geometry of this Fefferman manifold $F$ is equivalent to the CR geometry of $M$ [BDS]. In particular, since the set of null geodesics of a pseudo-Riemannian manifold is conformally invariant, all null geodesics of $F$ give rise (via the projection map) to CR-invariant curves on $M$.

All chains of $M$ are projections of null geodesics of $F$ [BDS]. However, not all null geodesics project to chains. For instance, the fibers of $F$ over $M$ are null geodesics (as we shall see in the following), and thus these project to points. If the Levi form of $M$ is indefinite $(p, q \geq 1)$, there are also other null geodesics, with nontrivial projections, which do not project to chains. Nevertheless, these projected curves are CR invariants of $M$. In order to study these new curves, we must first discuss the Fefferman correspondence in some detail. 
Let $M$ be a nondegenerate CR manifold of dimension $2 n+1$ and Levi signature $p, q$. Let $C=K^{*} / \mathbb{R}^{+}$be the quotient of the canonical bundle $K$ of $M$ with is zero section removed by the natural action of $\mathbb{R}^{+} ; C$ is a circle bundle over $M$. The Fefferman manifold $F$ of $M$ is obtained by equipping $C$ with a canonical restricted conformal class of pseudo-Riemannian metrics of signature $2 p+1,2 q+1$. We will briefly sketch a construction of this conformal class due to $J$. Lee $[\mathrm{L}]$. This construction will give a particular representative of the class in terms of a choice of pseudo-Hermitian structure on $M$; the restricted conformal class of this representative metric is invariant under change of pseudo-Hermitian structure.

Choose a pseudo-Hermitian structure on $M$; that is, choose a (real) one-form $\theta$ on $M$ annihilating the hyperplane field $\mathscr{H}$. Let $T$ be the unique vector field on $M$ so that $\theta(T)=1$ and $T\lrcorner d \theta=0$. Choose $n$ complex one-forms $\theta^{\alpha}$ on $M$ so that the $\theta^{\alpha}$, restricted to $T_{1,0}$, form a basis of $T^{1,0}$, and so that $\theta^{\alpha}(T)=0$.

These choices determine a (local) section $\zeta_{0}=\theta \wedge \theta^{1} \wedge \cdots \wedge \theta^{n}$ of $K^{*}$, and thus also a section of $C$. Let $\gamma$ be the variable $(\bmod 2 \pi)$ on the fibers of $C$

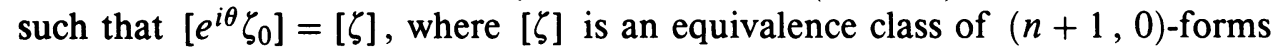
at a point of $M$. Let $K$ be the vertical (that is, tangent to the fibers) vector field such that $d \gamma(K)=1$.

For a CR manifold $M$ of Levi signature $p, q$, the Fefferman metric on $C$ will be the pseudo-Riemannian metric $d s^{2}=\mathrm{L}_{\theta}+2 \theta \sigma$ of signature $(2 p+$ $1,2 q+1)$, where $\sigma$ is a canonically specified real one-form on $C$ with $\sigma(K) \neq$ 0 . To construct $\sigma$, first let $\eta$ be an $n$-form on $C$ satisfying $\zeta=\theta \wedge \eta, \bar{T}\lrcorner \eta=0$ for any lift $\bar{T}$ of $T$ to $C$. Lee shows that $\eta$ is unique. The one-form $\sigma$ is then chosen to satisfy

$$
\begin{aligned}
d \zeta & =i(n-2) \sigma \wedge \zeta, \\
\sigma \wedge d \eta \wedge \bar{\eta} & =\operatorname{Tr}(d \sigma) i \sigma \wedge \theta \wedge \eta \wedge \bar{\eta} .
\end{aligned}
$$

Lee shows that conditions (L1) and (L2) determine $\sigma$ uniquely, and that the resulting metric $d s^{2}=\mathrm{L}_{\theta}+2 \theta \sigma$ agrees with the one defined by Fefferman on nondegenerate $\mathrm{CR}$ hypersurfaces in $\mathbb{C}^{n+1}$.

Sparling has shown (see $[\mathrm{S}, \mathrm{G}]$ ) that a pseudo-Riemannian manifold $F^{2 n+2}$ of signature $(2 p+1,2 q+1)$ is locally the Fefferman manifold of some nondegenerate CR manifold $M^{2 n+1}$ of Levi signature $(p, q)$ if and only if $F$ admits a null Killing vector field $K$ satisfying

(1) $K\lrcorner$ Weyl $=0, K\lrcorner \mathrm{Sch}=0$,

(2) $\operatorname{Ric}(K, K)>0$,

where Weyl is the Weyl tensor of $F$, Sch is the Schouten tensor, and Ric is the Ricci tensor. These conditions are invariant under conformal changes of metric of $F$ for which $K$ is a Killing field of both the new and old metrics. In the presence of condition $(1), \operatorname{Ric}(K, K)$ is actually constant; we scale $K$ so that $\operatorname{Ric}(K, K)=2 n$.

We have asserted that the various $C R$ invariants of $M$ may be recovered from the conformal geometry of $F$. Choose a point $x \in M$ and a point $\hat{x} \in F$ with $\pi(\hat{x})=x$, where $\pi: F \rightarrow M$ is the obvious projection map; then, in particular, $\mathscr{H}_{x}$ is the projection of $K_{x}^{\perp}=\left\{X \in T_{\hat{x}} F:\langle K, X\rangle=0\right\} ; \mathscr{J}$ is the projection of the restriction of $\nabla K$ to $K^{\perp}$; and the Levi form of $M$ is the 
projection of the restriction of the metric of $F$ to $K^{\perp}$. (For more details, see [Ko].)

We must make one crucial observation about the curvature of $F$. As a consequence of Sparling's condition $K\lrcorner$ Weyl $=0$, we find that

$$
\langle\mathrm{R}(X, K) K, Y\rangle=\frac{1}{2 n} \operatorname{Ric}(K, K)\langle X, Y\rangle \quad \text { for } X, Y \in K^{\perp} .
$$

If we normalize $K$ so that $\operatorname{Ric}(K, K)=2 n$, then $\langle\mathrm{R}(X, K) K, Y\rangle=\langle X, Y\rangle$ for $X, Y \in K^{\perp}$. Equivalently, we may say that $\mathrm{R}(X, K) K=X(\bmod K)$. This fact will be of central importance in several of our upcoming calculations.

\section{BASIC PROPERTIES OF NULL-CHAINS}

If $M$ is a strictly pseudoconvex CR manifold (of signature $(0, q)$ ), its Fefferman manifold $F$ is of Lorentz signature $(-,+, \ldots,+)$. Let $x \in M$ and let $\hat{x}$ be a point of $F$ with $\pi(\hat{x})=x$. In this signature, the set of all null vectors in $T_{\hat{x}} F$ intersects $K_{\hat{x}}^{\perp}$ in a single line, the span of $K_{\hat{x}}$.

But, if the Levi form of $M$ is strictly indefinite $(p, q \neq 0)$, then there are null vectors $\hat{V}$ in $T_{\hat{x}} F$ contained in $K_{\hat{x}}^{\perp}$ which are not multiples of $K_{\hat{x}}$. These vectors project to vectors $V$ in the holomorphic tangent space $\mathscr{H}_{x}=\pi_{*}\left(K_{\hat{x}}^{\perp}\right)$ of $M$ at $x$ which are null directions of the Levi form: $\mathrm{L}(V, V)=0$. Conversely, any $V \in \mathscr{H}_{x}$ with $\mathrm{L}(V, V)=0$ lifts to a null vector $\hat{V} \in K^{\perp}$, but this lift is not unique. For if $\hat{V}+\alpha K$ is another lift of $V$, then

$$
\langle\hat{V}+\alpha K, \hat{V}+\alpha K\rangle=\langle\hat{V}, \hat{V}\rangle+2 \alpha\langle\hat{V}, K\rangle+\alpha\langle K, K\rangle=0
$$

and

$$
\langle\hat{V}+\alpha K, K\rangle=\langle\hat{V}, K\rangle+\alpha\langle K, K\rangle=0
$$

as well. Thus any vector $V \in \mathscr{H}_{x}$ which is a null vector of the Levi form $(\mathrm{L}(V, V)=0)$ has a one-parameter family of lifts to null vectors in $K^{\perp}$. By projecting the null geodesics with these initial directions onto $M$, we obtain a one-parameter family of CR-invariant curves tangent to $V$ at $x$. By the conservation lemma (which says that along any geodesic $\beta,\left\langle\beta^{\prime}, K\right\rangle$ is constant), each of these curves remains tangent to $\mathscr{H}=\pi\left(K^{\perp}\right)$ at all points of the curve.

Definition. Let $M$ be a nondegenerate CR manifold. Let $F$ be its Fefferman manifold, and let $K$ be the vertical Killing vector field on $F$. The projection to $M$ of any nonvertical null geodesic of $F$ which is perpendicular to $K$ is called a null-chain.

Proposition 3.1. Through any point $x \in M$, in any direction $V \in \mathscr{H}_{x}$ with $\mathrm{L}(V, V)=0$, there passes a one-parameter family of distinct null-chains.

Proof. We must show that the null-chains through $x$ tangent to $V$ are distinct. Let $\hat{V}$ be any (fixed) lift of $V$ to $T_{\hat{x}} F$. Let $\beta(t)$ be the null geodesic with $\beta(0)=\hat{x}, \beta^{\prime}(0)=\hat{V}$ and extend $\hat{V}$ along $\beta$ by setting $\hat{V}=\beta^{\prime}(t)$. Let $B$ be the 2-surface swept out by $\beta(t)$ under the action of $K$, and extend $\hat{V}$ along $B$ accordingly. We want to show that geodesics through $\hat{x}$ with initial vectors $\hat{V}+\alpha K($ where $\alpha \neq 0)$ do not lie in $B$.

We compute

$$
\begin{aligned}
\nabla_{(\hat{V}+\alpha K)}(\hat{V}+\alpha K) & =\nabla_{\hat{V}} \hat{V}+2 \nabla_{\hat{V}} K+\alpha \nabla_{K} \hat{V}+\hat{V}(\alpha) K+\alpha^{2} \nabla_{K} K \\
& =2 \alpha \nabla_{K} \hat{V}+\hat{V}(\alpha) K
\end{aligned}
$$


where $\alpha$ is some function $\alpha: F \rightarrow \mathbb{R}$ with $K(\alpha)=0$. In order for $\hat{V}+\alpha K$ to be pregeodesic, this vector must lie in the span of $\hat{V}$ and $K$ at $\hat{x}$. But if $2 \alpha(\hat{x}) \nabla_{K} \hat{V} \in \operatorname{span}(\hat{V}, K)$ at $\hat{x}$, for $\alpha(\hat{x}) \neq 0$, then $V$ must be a real eigenvector of the CR structure tensor $\mathscr{J}: \mathscr{H} \rightarrow \mathscr{H}$. Since no such eigenvector exists, $B$ contains no geodesics through $\hat{x}$ other than $\beta$ and the vertical geodesic. Thus null geodesics through $\hat{x}$ with initial vectors $\hat{V}+\alpha K, \alpha \neq 0$, have distinct projections to null-chains of $M$.

Proposition 3.2. Every null geodesic of $F$ projects to a point, a null-chain, or a chain of $M$.

Proof. If $\beta(t)$ is a geodesic of $F$, where $F$ is equipped with a particular metric $\langle$,$\rangle in the conformal class of Fefferman metrics arising from the CR$ structure of $M$, then $\left\langle\beta^{\prime}(t), K\right\rangle$ is constant along $K$. (This is the well-known conservation lemma.) For

$$
\beta^{\prime}\left\langle\beta^{\prime}, K\right\rangle=\left\langle\nabla_{\beta^{\prime}} \beta^{\prime}, K\right\rangle+\left\langle\beta^{\prime}, \nabla_{\beta^{\prime}} K\right\rangle=0+0,
$$

since $\beta$ is a geodesic and $K$ is Killing. Thus the null geodesics of $F$ can be divided into three classes: vertical, nonvertical and perpendicular to $K$, and not perpendicular to $K$. This classification of null geodesics is conformally invariant. The three classes project to points, null-chains, and chains, respectively.

Proposition 3.3. Null-chains are pointwise limits of chains.

Proof. Null geodesics perpendicular to $K$ are limits of nonperpendicular null geodesics, and the projection map is continuous.

The following proposition tells us that null-chains are "easier to come by" than chains, in the sense that it is not necessary to complete the construction to the Fefferman metric in order to compute them.

Proposition 3.4. Let $\tilde{\mathrm{g}}$ be any metric on the underlying space of $F$, of the form

$$
\tilde{\mathrm{g}}=\mathrm{L}_{\theta}+2 \theta \sigma
$$

for some choice of pseudo-Hermitian structure $\theta$ on $M$ and for some one-form $\sigma$ on $F$ with $\sigma(K) \equiv 1$. If $\tilde{\mathrm{g}}$ satisfies normalization (L1) of $\S 3$, then the null geodesics of $\tilde{\mathbf{g}}$ which are perpendicular to the fibers of $F$ project to the null-chains of $M$.

Proof. Given $\theta$ of $M$, equation (L1) determines $\tilde{\mathrm{g}}$ up to the addition of a multiple of $\theta^{2}$ [L]. If $\mathrm{g}$ is the representative metric of the Fefferman conformal class constructed using this choice of $\theta$, then $\tilde{\mathrm{g}}-\mathrm{g}=\mu \theta^{2}$ for some function $\mu$ with $k(\mu)=0$, where $K$ is the vertical Killing field for both $\mathrm{g}$ and $\tilde{\mathrm{g}}$.

Recall the formula for the Levi-Civita connection:

$$
\begin{aligned}
2\left\langle\nabla_{X} Y, Z\right\rangle= & X\langle Y, Z\rangle+Y\langle Z, X\rangle-Z\langle X, Y\rangle \\
& +2\{\langle Z,[X, Y]\rangle+\langle Y,[Z, X]\rangle-\langle X,[Y, Z]\rangle\} .
\end{aligned}
$$

Now, let $X$ be a vector field on $F$ with $\theta(X) \equiv 0$. Then $X\lrcorner \mathrm{g} \equiv X\lrcorner \tilde{\mathrm{g}}$, and in particular $\mathrm{g}(X, K)=\tilde{\mathrm{g}}(X, K) \equiv 0$. So, for any vector field $Z$ on $F$,

$$
\begin{aligned}
\tilde{\mathbf{g}}\left(\nabla_{X} X, Z\right) & =X \tilde{\mathbf{g}}(X, Z)+2 \tilde{\mathbf{g}}(X,[Z, X])-2 Z \tilde{\mathbf{g}}(X, X) \\
& =X \mathbf{g}(X, Z)+2 \mathbf{g}(X,[Z, X])-2 Z \mathbf{g}(X, X) \\
& =g\left(\nabla_{X} X, Z\right) .
\end{aligned}
$$


Thus $\mathrm{g}$ and $\tilde{\mathrm{g}}=\mathrm{g}+\mu \theta^{2}$ have the same geodesics perpendicular to $K$. (Only the null geodesics are invariant under changes of $\theta$, however.)

\section{NULL-CHAINS ON THE INDEFINITE HYPERQUADRICS}

The chains on the hyperquadric of signature $(p, q)$ in $\mathbb{C}^{p+q+1}$,

$$
Q_{p, q}=\left\{\left(z_{0}, \ldots, z_{p+q}\right): \Im z_{0}=\left|z_{1}\right|^{2}+\cdots+\left|z_{p}\right|^{2}-\left|z_{p+1}\right|^{2}-\cdots-\left|z_{p+q}\right|^{2}\right\},
$$

are the curves defined by intersecting $Q_{p, q}$ with affine complex lines of the ambient complex space (when this intersection defines a real curve). Thus all chains on $Q_{p, q}$ are circles (perhaps passing through the point at infinity) which lie in some $\mathbb{C}^{1}$ of $\mathbb{C}^{p+q+1}$.

The null chains on $Q_{p, q}$ may be found equally explicitly. If neither $p$ nor $q$ is zero, there are affine complex lines of the ambient $\mathbb{C}^{p+q+1}$ which are entirely contained in $Q_{p, q}$; for these complex lines, the intersection with $Q_{p, q}$ does not define a chain. The null-chains on $Q_{p, q}$ are all the circles (perhaps passing through infinity) contained in all the complex lines which are entirely contained in $Q_{p, q}$, as we shall see below.

In order to calculate the null-chains on $Q_{p, q}$, it will be useful to regard $Q_{p, q}$ as a hypersurface in $\mathbb{C P}^{n+1}$ rather than $\mathbb{C}^{n+1}$, where $p+q=n$. Thought of in this way, $Q_{p, q}$ is the complex projectivization of the solution set in $\mathbb{C}^{n+2}$ of the equation

$$
\left|z_{1}\right|^{2}+\cdots+\left|z_{p+1}\right|^{2}-\left|z_{p+2}\right|^{2}-\cdots-\left|z_{n+2}\right|^{2}=0 .
$$

Identifying $\mathbb{C}^{n+2}$ with $\mathbb{R}^{2 n+4}$ by $z_{j}=x_{j}+i y_{j}$, we see that this solution set is the lightcone

$$
\begin{aligned}
L C=\left\{(x, y) \in \mathbb{R}^{2 n+4}: x_{1}^{2}+y_{1}^{2}+\cdots\right. & +x_{p+1}^{2}+y_{p+1}^{2} \\
& \left.-x_{p+2}^{2}-y_{p+2}^{2}-\cdots-x_{n+2}^{2}-y_{n+2}^{2}=0\right\}
\end{aligned}
$$

of $\mathbb{R}^{2 n+4}$ with the pseudo-Euclidean metric of signature $(2 p+2,2 q+2)$. If we now take the real projectivization of $L C$, we obtain a $(2 n+2)$-dimensional real quadric $F_{p, q}$, which may be viewed as a circle bundle over $Q_{p, q}$ by the restirction of the Hopf fibration $S^{1} \rightarrow \mathbb{R P}^{2 n+3} \rightarrow \mathbb{C P}^{n+1}$. If the vector field tangent to the (isometric) $S^{1}$-action on $F_{p, q}$ is labelled $K$, then $F_{p, q}$ satisfies Sparling's conditions. Thus $F_{p, q}$ is the Fefferman manifold of $Q_{p, q}$.

Any $\mathbb{C P}^{1}$ in $\mathbb{C} \mathbb{P}^{n+1}$ is the projection of a vertical $\mathbb{R} \mathbb{P}^{3}$ in $\mathbb{R} \mathbb{P}^{2 n+3}$. If a given $\mathbb{C P}^{1}$ in $\mathbb{C P}^{n+1}$ is not wholly contained in $Q_{p, q}$, then its intersection with $Q_{p, q}$ is a chain. But if it is contained in $Q_{p, q}$, the $\mathbb{C P}^{1}$ is the projection of a vertical $\mathbb{R P}^{3}$ wholly contained in $F_{p, q} ;$ this $\mathbb{R} \mathbb{P}^{3}$ is necessarily totally null and perpendicular to the fibers of $F_{p, q}$. Moreover, this $\mathbb{R P}^{3} \subset F_{p, q} \subset \mathbb{R \mathbb { P } ^ { 2 n + 3 }}$ is a totally geodesic submanifold of $\mathbb{R} \mathbb{P}^{2 n+3}$, and thus also of $F_{p, q}$. The geodesic "great circles" of this $\mathbb{R P}^{3}$ (all of which are null geodesics of $F_{p, q}$ ) project to all circles of the corresponding $\mathbb{C P}^{1} \subset Q_{p, q}$. Thus we see that the null-chains of $Q_{p, q}$ are all the circles in all the $\mathbb{C P}^{1}$ 's contained in $Q_{p, q}$.

Let us make two observations about the null-chains on $Q_{p, q}$. First, any two points of $Q_{p, q}$ can be joined by either a chain or a null-chain. The two points determine a complex line in $\mathbb{C}^{n+1}$. This complex line either intersects $Q_{p, q}$ in 
a chain connecting the points, or else it is entirely contained in $Q_{p, q}$, in which case any circle in the complex line which passes through the two points may be taken as a null-chain connecting them.

Second, any two points which are joined by a null-chain, are joined by a oneparameter family of null-chains. If the two points are joined by a null-chain, then the complex line they determine in $\mathbb{C}^{n+1}$ must be contained in $Q_{p, q}$. Within this $\mathbb{C}^{2} \subset Q_{p, q}$, the two points determine a one-parameter family of circles.

It will be the object of the next two sections to show that these connectivity properties for the chains and null-chains on the hyperquadric in fact hold locally for any nondegenerate CR manifold.

\section{VARIATION THEOREM FOR NULL-CHAINS}

On the face of it, one might expect the set of points reached by null-chains from a fixed point $x$ of a CR manifold of Levi signature $(p, q)$ to be a set described by $2 p+2 q$ parameters, since there is a one-parameter family of nullchains in each of the $\left(S^{2 p-1} \times S^{2 q-1}\right)$ 's worth of eligible directions. However, on the hyperquadric $Q_{p, q}$, we saw that the set of such points was in fact a cone on $S^{2 p-1} \times S^{2 q-1}$, and thus this set can be described by $2 p+2 q-1$ parameters, one less than expected. One might ask whether this behavior of the null-chains of $Q_{p, q}$ is due to the fact that all null-chains of $Q_{p, q}$ are contained in complex submanifolds (namely $\mathbb{C}^{1}$ 's) of $Q_{p, q}$, whereas indefinite CR manifolds need not contain any complex submanifold in general [Br]. It turns out that this one-parameterless phenomenon is general; in fact, two nearby points which lie on a null-chain, lie on a one-parameter family of null-chains.

Theorem 6.1. Let $M$ be a nondegenerate CR manifold of strictly indefinite Levi signature. Then any two sufficiently nearby points $x, y \in M$ which lie on $a$ null-chain lie on a continuous one-parameter family of null-chains.

The proof of this theorem will take place in the Fefferman manifold $F$ of $M$, so we must restate the theorem in $F$.

Theorem 6.2. Let $\gamma(t)$ be the fiber over $x \in M$. Let $\sigma(s)$ be a null geodesic of $F$ with $\sigma(0)=\gamma(0)$ and $\left\langle\sigma^{\prime}(0), \gamma^{\prime}(0)\right\rangle=0$. Let $W_{s_{0}}(t, s)$ be a variation of $\sigma$ through geodesics $W_{s_{0}, t}(s)$ from $\sigma\left(s_{0}\right)$ to $\gamma$, with $W_{s_{0}}\left(t, s_{0}\right)=\sigma\left(s_{0}\right)$, $W_{s_{0}}(0, s)=\sigma(s)$, and $W_{s_{0}}(t, 0)=\gamma(t) ; W_{s_{0}}$ is defined for $s \in\left[0, s_{0}\right]$ and $t \in\left[0, t_{0}\right]$, provided $s_{0}$ and $t_{0}$ are small enough. Then the variation $W_{s_{0}}$ of $\sigma$ is through null geodesics perpendicular to $\gamma$.

Note. The existence and uniqueness of the geodesics $W_{s_{0}, t}(s)$ is assured by working in a convex normal coordinate neighborhood of $\gamma(0)$ in $F$.

We will need the following lemma:

Lemma 6.3. Let $\gamma(t)$ be a null geodesic in a pseudo-Riemannian manifold $X^{m+2}$. Assume that $\langle\mathrm{R}(X, T) T, Y\rangle=\langle X, Y\rangle$ for all $X, Y$ perpendicular to $\gamma$, where $T=\gamma^{\prime}$. Let $J$ be any Jacobi field along $\gamma$ with $J(0)=0,\left.\langle J, T\rangle\right|_{t_{0}}=0$, $\left.\langle J, J\rangle\right|_{t_{0}}=0$, and $J\left(t_{0}\right) \neq 0$ for some fixed $t_{0} \in(0, \pi)$. Then $\langle J, J\rangle=0$ for all $t \in[0, \pi]$.

Proof of Lemma 6.3. Choose a frame $Y_{1}, \ldots, Y_{n}, T$ of $\left(\gamma^{\prime}\right)^{\perp}$ at $\gamma(0)$ with $\left\langle Y_{i}, Y_{j}\right\rangle= \pm \delta_{i j},\left\langle Y_{i}, T\right\rangle=0 \forall i$. Extend $Y_{1}, \ldots, Y_{n}$ along $\gamma$ by parallel 
transport. Note that $J(t) \in\left(\gamma^{\prime}\right)^{\perp}$ for all $t$, since

$$
\langle J, T\rangle=\langle\mathrm{R}(J, T) T, T\rangle \equiv 0
$$

by our assumption.

Write $J=a_{1}(t) Y_{1}+\cdots+a_{n}(t) Y_{n}+a_{0}(t) T$. Then

$$
\begin{aligned}
\nabla_{T} J & =a_{1}^{\prime} Y_{1}+\cdots+a_{n}^{\prime} Y_{n}+a_{0}^{\prime} T, \\
\nabla_{T} \nabla_{T} J & =a_{1}^{\prime \prime} Y_{1}+\cdots+a_{n}^{\prime \prime} Y_{n}+a_{0}^{\prime \prime} T .
\end{aligned}
$$

By Jacobi's equation $\nabla_{T} \nabla_{T} J+\mathrm{R}(J, T) T=0$ and our curvature assumption, we have

$$
\nabla_{T} \nabla_{T} J=-J(\bmod T) .
$$

So $a_{i}^{\prime}=-a_{i}$ for $i=1, \ldots, n, t \in[0, \pi]$. Since $J(0)=0$, we see that $a_{i}(t)=A_{i} \sin t$ for $i=1, \ldots, n$, where $A_{i}=a_{i}(0)$.

Let us assume (without loss of generality) that the frame $Y_{1}, \ldots, Y_{n}, T$ was chosen so that $J\left(t_{0}\right)=c\left(Y_{1}-Y_{2}\right)$, where $\left\langle Y_{1}, Y_{1}\right\rangle+\left\langle Y_{2}, Y_{2}\right\rangle=0$. Then $J(t)=c \sin t Y_{1}-c \sin t Y_{2}(\bmod T)$ for all $t \in[0, \pi]$. Thus

$$
\langle J, J\rangle=c^{2} \sin ^{2} t\left(\left\langle Y_{1}, Y_{1}\right\rangle+2\left\langle Y_{1}, Y_{2}\right\rangle+\left\langle Y_{2}, Y_{2}\right\rangle\right) \equiv 0 \quad \text { for all } t \in[0, \pi]
$$

We note also that $\left\langle\nabla_{T} J, \nabla_{T} J\right\rangle=0$ on $[0, \pi]$.

Corollary 6.4. Let $X, \gamma$ be as in Lemma 6.3. Let $J$ be a Jacobi field along $\gamma$, with $J(0)=0$ and $\left.\langle J, T\rangle\right|_{t_{0}}=0, J\left(t_{0}\right) \neq 0$ for some $t_{0} \in(0, \pi)$. Then the sign of $\langle J, J\rangle$ is constant on $(0, \pi)$.

Proof of Lemma 6.2. We will be forced to construct the variation $W_{s_{0}}$ in a rather roundabout manner, in order to see that it is a variation of $\sigma$ through null geodesics. We shall first construct a preliminary variation $V_{t_{1}}(t, s)$ of $\gamma$ through geodesics from $\gamma\left(t_{1}\right)$ to $\sigma$ for a fixed $t_{1} \in\left(0, t_{0}\right) . V_{t_{1}}$ will be defined for $t \in\left[0, t_{1}\right]$ and for $s \in\left[0, s_{0}\right] . \quad V_{t_{1}}$ will be shown to be a variation of $\gamma$ through null geodesics. Thus the unique geodesic from $\sigma\left(s_{0}\right)$ to $\gamma\left(t_{1}\right)$ is null, for each $t_{1} \in\left(0, t_{0}\right)$.

Next, by letting $t_{1}$ vary, we construct the variation $W_{s_{0}}$, which we now know to be through null geodesics. Then it will only remain to be shown that the geodesics $W_{s_{0}},{ }_{t}(s)$ are perpendicular to $\gamma$.

To begin, fix a $t_{1} \in\left[0, t_{0}\right]$, and let $V_{t_{1}}(t, s)$ be a variation of $\gamma$ through geodesics from $\gamma\left(t_{1}\right)$ to $\sigma$, with $V_{t_{1}}(0, s)=\sigma(s), V_{t_{1}}\left(t_{1}, s\right)=\gamma\left(t_{1}\right) \quad \forall s$, $V_{t_{1}}(t, 0)=\gamma(t)$, and $V_{t_{1}}(t, s)=V_{t_{1} s}(t)$ geodesic for each fixed $s$. Let $T, S$ be the variation vector fields corresponding to the $t, s$ variables. Then $\nabla_{T} T \equiv 0$; $\nabla_{S} S=0$ if $t=0 ;\langle S, T\rangle=0$ at $t=0, s=0$ and at $t=t_{1}, s=0$; $[T, S] \equiv 0$; and $T=K$ along $\gamma(s=0)$. Our aim is to show that the geodesics $V_{t_{1}},{ }_{s}(t)$ are null, that is, that $\langle T, T\rangle \equiv 0$.

Since $\langle T, T\rangle=\langle K, K\rangle=0$ along $\gamma$, we must show that $\langle T, T\rangle$ is constant in $s$. Note that

$$
\begin{aligned}
S\langle T, T\rangle & =2\left\langle\nabla_{S} T, T\right\rangle=2\left\langle\nabla_{T} S, T\right\rangle \\
& =2 T\langle S, T\rangle+2\left\langle S, \nabla_{T} T\right\rangle=2 T\langle S, T\rangle .
\end{aligned}
$$


So we may prove that $\langle T, T\rangle \equiv 0$ by proving that $\langle S, T\rangle \equiv 0$. We compute

$$
\begin{aligned}
T(T\langle S, T\rangle) & =T\left\langle\nabla_{T} S, T\right\rangle+T\left\langle\nabla_{T} T, S\right\rangle \\
& =\left\langle\nabla_{T} \nabla_{T} S, T\right\rangle+\left\langle\nabla_{T} S, \nabla_{T} T\right\rangle+0 \\
& =\langle R(T, S) T, T\rangle+0 \\
& \equiv 0 \quad \text { for all } t \text { and } s .
\end{aligned}
$$

Here we have used Jacobi's equation and the fact that $\nabla_{T} T \equiv 0$ in the last equation. Thus $\langle S, T\rangle$ is a linear function of $t$ for each fixed $s$. Since $\langle S, T\rangle=0$ at $t=t_{1}$ for all $s$, we must now compute $\langle S, T\rangle$ at $t=0$ for all $s$, that is, along $\sigma$.

We will now show that $\langle S, T\rangle$ is a linear function of $s$ along $\sigma$ with $\langle S, T\rangle=S\langle S, T\rangle=0$ at $\sigma(0)$. Along $\sigma$, we calculate

$$
\begin{aligned}
S(S\langle S, T\rangle) & =S\left\langle S, \nabla_{S} T\right\rangle=S\left\langle S, \nabla_{T} S\right\rangle \\
& =\left\langle\nabla_{S} S, \nabla_{T} S\right\rangle+\left\langle S, \nabla_{S} \nabla_{T} S\right\rangle \\
& =\langle\operatorname{R}(S, T) S, S\rangle \\
& \equiv 0 \quad \text { for all } s .
\end{aligned}
$$

Here we have used $\nabla_{S}=0$ along $\sigma$. Thus $\langle S, T\rangle$ is a linear function of $s$ along $\sigma$. We have $\langle S, T\rangle=0$ at $\sigma(0)$ by construction. To compute $S\langle S, T\rangle$ at $\sigma(0)$, write

$$
S\langle S, T\rangle=\left\langle\nabla_{S} S, T\right\rangle+\left\langle S, \nabla_{S} T\right\rangle=0+\left\langle S, \nabla_{T} S\right\rangle=\frac{1}{2} T\langle S, S\rangle .
$$

So $S\langle S, T\rangle$ at $s=0, t=0$ (i.e., $\sigma(0)$ ) equals $\frac{1}{2} T\langle S, S\rangle$ at $s=0, t=0$ (i.e., $\gamma(0))$. By Lemma V, $\langle S, S\rangle \equiv 0$ along $\gamma$, so $T\langle S, S\rangle=0$ at $s=0, t=0$. We may now conclude that $\langle S, T\rangle \equiv 0$ along $\sigma(t=0)$.

Now we know that $\langle S, T\rangle$ is a function linear in $t$ for each $s$, and that $\langle S, T\rangle=0$ for $t=0, \forall s$. Thus $\langle S, T\rangle \equiv 0$, and so $\langle T, T\rangle \equiv 0$ as in the first paragraph. Thus the variation $V_{t_{1}}(t, s)$ of $\gamma$ is through null geodesics from $\gamma\left(t_{1}\right)$ to $\sigma$.

We now know that, for $t_{1}$ and $s$ small enough, the unique geodesic from $\gamma\left(t_{1}\right)$ to $\sigma(s)$ is null. We are now ready to construct the variation we seek. Let $W_{s_{0}}(t, s)$ be a variation of $\sigma$ through geodesics from $\sigma\left(s_{0}\right)$ to $\gamma$, with $W_{s_{0}}(0, s)=\sigma(s), W_{s_{0}}(t, 0)=\gamma(t), W_{s_{0}}=\sigma\left(s_{0}\right)$ for all $t$, and $W_{s_{0}}=W_{s_{0}, t}(s)$ geodesic for each fixed $t$. Let $\tilde{T}, \tilde{S}$ be the variation vector fields corresponding to the $t, s$ variables. Then $\nabla_{\tilde{S}} \tilde{S} \equiv 0, \nabla_{\tilde{T}} \tilde{T}=0$ if $s=0,[\tilde{T}, \tilde{S}] \equiv 0$, $\langle\tilde{T}, \tilde{T}\rangle=0$ if $s=0$, and $\langle\tilde{S}, \tilde{T}\rangle=0$ at $t=0, s=0$ and $t=0, s=s_{0}$. We also know by the preceding argument that $\langle\tilde{S}, \tilde{S}\rangle \equiv 0$ for all $t$ and $s$, since the unique geodesic from $\sigma\left(s_{0}\right)$ to $\gamma(t)$ is null for all $s, t$ sufficiently small; thus $W_{s_{0}}$ is a variation of $\sigma$ through null geodesics.

We must now prove that these geodesics $W_{s_{0}, t}(s)$ are perpendicular to $\gamma$; that is, we need $\langle\tilde{S}, \tilde{T}\rangle=0$ if $s=0$, for all $t$. We will in fact show that $\langle\tilde{S}, \tilde{T}\rangle \equiv 0$. Compute

$$
\begin{aligned}
\tilde{S}\langle\tilde{S}, T\rangle & =\left\langle\nabla_{\tilde{S}} \tilde{S}, T\right\rangle+\left\langle\tilde{S}, \nabla_{\tilde{S}} \tilde{T}\right\rangle=0+\left\langle\tilde{S}, \nabla_{\tilde{T}} \tilde{S}\right\rangle \\
& =\frac{1}{2} \tilde{T}\langle\tilde{S}, \tilde{S}\rangle \equiv 0 \text { for all } s \text { and } t .
\end{aligned}
$$

Thus $\langle\tilde{S}, \tilde{T}\rangle$ is a constant function of $s$ for each $t$. But $\langle\tilde{S}, \tilde{T}\rangle=0$ for $s=s_{0}$, for all $t$. Therefore $\langle S, T\rangle \equiv 0$ for all $s$ and $t$, and the variation $W_{s_{0}}$ of $\sigma$ is through null geodesics perpendicular to $\gamma$, as required. 


\section{CONNECTIVITy TheOREM}

We now turn our attention to the task of proving that any point $x$ of a nondegenerate CR manifold $M$ can be joined to every sufficiently nearby point $y$ by either a chain or a null chain.

The idea of the proof is to show that, for a point $\hat{x}$ in the Fefferman manifold $F$ of $M$ with $\pi(\hat{x})=x$, the lightcone of $\hat{x}$ is shaped so that the projection map $\pi: F \rightarrow M$ carries a suitable portion of it onto a neighborhood of $x$ in $M$. Since the lightcone of $\hat{x}$ is the set of those points of $F$ which can be reached from $\hat{x}$ by a null geodesic, this amounts to showing that $x$ can be joined to each point of the neighborhood in $M$ by the projection of a null geodesic of $F$, i.e., by a chain or a null-chain.

We will work with a fixed representative metric of the restricted conformal class of Fefferman metrics on $F$, and by " $F$ " we will mean the Fefferman manifold with this choice of metric. Our results, of course, are preserved under (restricted) conformal change of metric. (For the sake of simplicity of exposition, we will actually work with a covering space of the subset $\pi^{-1}\left(N_{x}\right)$ of $F$, where $N_{x}$ is a neighborhood of $x$ in $M$, obtained by "unrolling" the circular fibers into lines. Since the theorem we wish to prove is of a local nature on $M$, no generality is lost.)

Let $\gamma(t)$ be the (affinely parametrized null geodesic) fiber over $x \in M$; let $\hat{x}=\gamma(0)$. We begin by recalling that $\mathrm{R}(X, K) K=X(\bmod K)$ for $X \in K^{\perp}$. By Jacobi's equation, a point $\hat{x}=\gamma(0)$ must have focal points of maximal order along $\gamma$ at $t= \pm \frac{\pi}{2}$. That is, all Jacobi fields $J$ along $\gamma$ with $\left.\left\langle J, \gamma^{\prime}\right\rangle\right|_{t=0}=0$ and $J^{\prime}(0)=0$ vanish simultaneously $\left(\bmod \gamma^{\prime}\right)$ at $t= \pm \frac{\pi}{2}$. Similarly, $\hat{x}=\gamma(0)$ has its first conjugate points along $\gamma$ at $t= \pm \pi$, and they are of maximal order.

A lemma (Lemma 7.2) will assure us that there is a normal (i.e., geodesically star-shaped) neighborhood of $\gamma(0)$ containing $\gamma([a, b])$ provided that $-\pi<$ $a<0<b<\pi$. In particular, there exists such a neighborhood containing $\gamma\left(\left[-\frac{\pi}{2}-\varepsilon, \frac{\pi}{2}+\varepsilon\right]\right)$ so long as $0<\varepsilon<\frac{\pi}{2}$. Working within such a neighborhood, we choose a plane $P^{2 n} \subset T_{\hat{x}} F$ of codimension 2 , such that $P \subset K^{\perp}$ and $K \notin P$. ( $P$ is a lift of the holomorphic tangent plane $H$ at $x$ in $M$.) Let $\Sigma_{0}=\exp _{\hat{x}} P ; \Sigma_{0}$ is a nondegenerate pseudo-Riemannian submanifold of $F$ of signature $(2 p, 2 q)$, where $(p, q)$ is the Levi signature of $M$.

We will first show that all points of $\pi\left(\Sigma_{0}\right) \subset M$ may be reached from $x$ by the projection of certain null geodesics in the lightcone $L C(\hat{x})$ of $\hat{x}$. Then, we will use an argument involving the topology of this lightcone to show that, in fact, every point in a neighborhood of $x$ lies in $\pi(L C(\hat{x}))$.

To show that $\pi\left(\Sigma_{0}\right) \subset \pi(L C(\hat{x}))$, we divide $\Sigma_{0}-\hat{x}$ into three regions (keeping in mind that each point of $\Sigma_{0}$ may be reached from $\hat{x}$ by a geodesic in $\Sigma_{0}$ ): region (1) consists of those points of $\Sigma_{0}$ which lie on a timelike geodesic from $\hat{x}=\gamma(0)$, region (2) consists of those points which lie on a spacelike geodesic from $\hat{x}$, and region (3) contains those points which lie on a null geodesic from $\hat{x}$. (If $M$ is strictly pseudo-convex, regions (1) and (3) will be empty.) $\Sigma_{0}$ is the disjoint union of these three regions with $\{\hat{x}\}$. Let $\Sigma_{t}$ be the translate of $\Sigma_{0}$ under the action of $K$ to an isometric copy containing $\gamma(t) ; \Sigma_{t}-\gamma(t)$ splits into three regions, which we will also call (1), (2), and (3), the translates of the corresponding regions in $\Sigma_{0}$. By Theorem 6.2 and Lemma 7.2, those points of $\Sigma_{t}$ in region (3) which are close enough to $\gamma(t)$ remain reachable from $\gamma(0)$ by 
null geodesics for all $t \in\left[-\frac{\pi}{2}-\varepsilon, \frac{\pi}{2}+\varepsilon\right]$, for $0<\varepsilon<\frac{\pi}{2}$.

The aim of Lemma 7.3 is to show that the fibers through points of regions (1) and (2) of $\Sigma_{0}$ are reached by null geodesics from $\hat{x}=\gamma(0)$ at some point also. That is, for each $y_{0} \in \Sigma_{0}$, there is an $r$ such that the translate $y_{r} \in \Sigma_{r}$ of $y_{0}$ lies on a null geodesic from $\gamma(0)=\hat{x}$. Actually, we will show that, for some $t \in\left(\frac{\pi}{2}, \pi\right)$, every point $y_{t} \in \Sigma_{t}$ near $\gamma(t)$ in region (1) is reachable from $\gamma(0)$ by a spacelike geodesic (rather than the timelike geodesic from $\gamma(0)$ to $y_{0}$ ), and vice versa for region (2). Because we work in a normal neighborhood of $\gamma(0)$, it follows that there is a null geodesic from $\gamma(0)$ to $y_{r}$ for some $r \in(0, t)$. Another null geodesic from $\gamma(0)$ to $y_{r}$ can be found for some other $r \in\left(t_{1}, 0\right)$, where $t_{1} \in\left(-\pi,-\frac{\pi}{2}\right)$.

Lemma 7.2 will be derived as a consequence of the following more general proposition.

Proposition 7.1. Let $X$ and $Y$ be locally compact Hausdorff spaces. Let $f$ : $X \rightarrow Y$ be a local homeomorphism, and let $A$ be a compact subset of $X$ such that $\left.f\right|_{A}$ is injective. Then there is an open subset $U$ of $X$ containing $A$ such that $\left.f\right|_{U}$ is a homeomorphism.

Proof. If no such open set $U$ containing $A$ exists, there must be sequences of points $\left\{x_{\alpha}\right\},\left\{y_{\alpha}\right\}$ of $X$ arbitrarily close to $A$ such that $f\left(x_{\alpha}\right)=\left(y_{\alpha}\right)$. Let $V$ be an open subset of $X$ containing $A$ such that the closure $\bar{V}$ of $V$ is compact. Within $\bar{V}$, choose convergent subsequences $x_{i} \rightarrow x$ and $y_{i} \rightarrow y$ with $x, y \in A$. Since $f$ is continuous, $f(x)=f(y)$.

If $x \neq y$, then $f$ is not injective on $A$. But if $x=y$, then $f$ is not one-toone on any neighborhood of $x$, and thus $f$ cannot be a local homeomorphism.

Thus no such sequences can exist, and there is some neighborhood $U$ of $A$ in $X$ such that $\left.f\right|_{U}$ is a homeomorphism.

Corollary (Lemma 7.2). Let $\gamma([a, b]), 0 \in(a, b)$, be a segment of a geodesic in a pseudo-Riemannian manifold $Y$, such that $\gamma([a, b])$ has no self-intersections and contains no point conjugate to $\gamma(0)$. Then there is a neighborhood $U$ of $\gamma([a, b])$ which is a normal neighborhood of $\gamma(0)$.

Proof. Apply the proposition with $X=T_{\gamma(0)} Y, A=\exp _{\gamma(0)}^{-1}(\gamma([a, b]))$, and $f=\exp _{\gamma(0)}$, to obtain a neighborhood $\tilde{U}$ of $\gamma([a, b])$ on which $\exp _{\gamma(0)}$ is invertible.

Then choose a neighborhood of $A=\exp _{\gamma(0)}^{-1}(\gamma([a, b]))$ contained in $\exp _{\gamma(0)}^{-1}(\tilde{U})$ which is star-shaped with respect to $0 \in T_{\gamma(0)} Y$; this is possible because $A$ is star-shaped with respect to 0 . The image of this star-shaped neighborhood is the required normal neighborhood $U$ of $\gamma(0)$ containing $\gamma([a, b])$.

Lemma 7.3. Let $F^{2 n+2}$ be the Fefferman manifold of a $C R$ manifold $M^{2 n+1}$ of Levi signature $(p, q)$. Let $\gamma(t)$ be the fiber over $x \in M$. Let $\Sigma_{0}$ be an $(n-2)$ surface of metric signature $(2 p, 2 q)$ near $\gamma(0)$ such that $\Sigma_{0}=\exp _{\gamma(0)}(P)$ for a 2n-plane $P \subset K^{\perp}$. Let $\Sigma_{t}$ be the translate of $\Sigma_{0}$ to $\gamma(t)$ under the action of the vertical Killing field $K$ on $F$. Let $\varepsilon \in\left(0, \frac{\pi}{2}\right)$, and let $a=\frac{\pi}{2}+\varepsilon$. Then, if $y \in \Sigma_{ \pm a}$ lies on a timelike geodesic from $\gamma( \pm a)$ (respectively), it lies on a spacelike geodesic from $\gamma(0)$, and if $y$ lies on a spacelike geodesic from $\gamma( \pm a)$, it lies on a timelike geodesic from $\gamma(0)$, for all $y$ in a neighborhood of $\gamma( \pm a)$ in $\Sigma_{ \pm a}$. 
Proof. Fix a real number $b$ such that $a<b<\pi$. By Lemma 7.2, there is a normal neighborhood $U$ of $\gamma(0)$ containing $\gamma([-b, b])$; from now on we will work only in $U$. Every point (in $U$ ) of $\Sigma_{t}$, for $t \in[-b, b]$, lies on a unique geodesic from $\gamma(0)$. In addition, since $\Sigma_{t}$ is a translate of $\Sigma_{0}=\exp _{\gamma(0)} P$, each point of $\Sigma_{t}$ lies on a unique geodesic from $\gamma(t)$. Let $(s, \theta)$ be geodesic polar coordinates on $\Sigma_{t}$, where $\theta \in S^{2 n-1}$.

For any $t \in[-b, b]$, and any $\theta \in S^{2 n-1}$, we may construct a variation $V(\tau, s, \theta)$ of $\gamma$ through geodesics from $\gamma(0)$ to the points of $\Sigma_{t}$ near $\gamma(t)$ whose angular coordinate is that $\theta$, and we may require that the curves $V(s)$, for fixed $\tau=t$, are geodesics lying in $\Sigma_{t}$ for each $\theta$. Let $T, S_{\theta}$ be the variation vector fields with respect to the variables $\tau, s$. We have $V(\tau)=\gamma(\tau)$ for $s=0,\left.S_{\theta}\right|_{\tau=0}=0, \nabla_{S_{\theta}} S_{\theta}=0$ for $\tau=t, \nabla_{T} T \equiv 0, T=K$ for $s=0$, $\left\langle S_{\theta}, T\right\rangle=0$ for $s=0$ (i.e., along $\gamma$ ), and $\left[S_{\theta}, T\right] \equiv 0$.

Now, if $\left\langle S_{\theta}, S_{\theta}\right\rangle>0$ for $s=0, \tau=t, \theta$ fixed, then the curve $\beta_{\theta}(s)=$ $\exp _{\gamma(t)}\left(s S_{\theta}\right)$ is spacelike, so the points $V(\tau=t, s, \theta)$ lie on a spacelike geodesic from $\gamma(t)$. If $\left\langle S_{\theta}, S_{\theta}\right\rangle<0$, the geodesic from $\gamma(t)$ is timelike; if $\left\langle S_{\theta}, S_{\theta}\right\rangle=0$, it is null.

We wish to show that if for small $s>0$ and $\frac{\pi}{2}<t<\frac{\pi}{2}+\delta$, the point $V(\tau=t, s, \theta)$ lies on a spacelike geodesic from $\gamma(t)$, then $S_{\theta}\langle T, T\rangle<0$ for that value of $\theta$, and for all $\tau$ and all small $s>0$. This will indicate that the geodesic $V(\tau)=V(\tau, s, \theta)$, for this $\theta$ and for any small fixed $s>0$, is timelike. (The argument that the geodesic to $V(\tau=t, s, \theta)$ from $\gamma(0)$ is spacelike if the one from $\gamma(t)$ is timelike is similar and will be omitted; the argument for $-\frac{\pi}{2}-\delta<t<-\frac{\pi}{2}$ may be obtained by revising the signs of $T$ and $\tau$.) We compute

$$
\begin{aligned}
S_{\theta}\langle T, T\rangle & =2\left\langle\nabla_{S_{\theta}} T, T\right\rangle=2\left\langle\nabla_{T} S_{\theta}, T\right\rangle \\
& =2 T\left\langle S_{\theta}, T\right\rangle-2\left\langle S_{\theta}, \nabla_{T} T\right\rangle=2 T\left\langle S_{\theta}, T\right\rangle .
\end{aligned}
$$

By Jacobi's equation, $T\left(T\left\langle S_{\theta}, T\right\rangle\right)=0$ for all $t$ and $s$, so $\left\langle S_{\theta}, T\right\rangle$ is a linear function of $\tau$ for each fixed $s$.

Since $S_{\theta}=0$ if $\tau=0$, and $\left\langle S_{\theta}, T\right\rangle=0$ if $\tau=t$, we conclude first that $\left\langle S_{\theta}, T\right\rangle \equiv 0$ for $s=0$ (along $\gamma$ ), and second that for $\tau \neq 0$ and $s \neq 0$ fixed, the sign of $\left\langle S_{\theta}, T\right\rangle$ is the same as that of $T\left\langle S_{\theta}, T\right\rangle$. So now we must show that $S_{\theta}\left\langle S_{\theta}, T\right\rangle>0$ at $\tau=t, s=0$.

To achieve this, recall that $T=K$ along $\gamma$. By Jacobi's equation,

$$
\begin{aligned}
\left\langle S_{\theta}, S_{\theta}\right\rangle & =-\left\langle\mathrm{R}\left(T, S_{\theta}\right) T, S_{\theta}\right\rangle=-\left\langle\nabla_{T} \nabla_{T} S_{\theta}, S_{\theta}\right\rangle \\
& =-T\left\langle\nabla_{T} S_{\theta}, S_{\theta}\right\rangle+\left\langle\nabla_{T} S_{\theta}, \nabla_{T} S_{\theta}\right\rangle .
\end{aligned}
$$

By the calculations in the proof of Lemma 6.3, we know that $\left\langle\nabla_{T} S_{\theta}, \nabla_{T} S_{\theta}\right\rangle=0$ at $t=\frac{\pi}{2}$, and thus

$$
\left.\left\langle S_{\theta}, S_{\theta}\right\rangle\right|_{\tau=\frac{\pi}{2}}=-\left.T\left\langle\nabla_{T} S_{\theta}, S_{\theta}\right\rangle\right|_{\tau=\frac{\pi}{2}}
$$

Moreover,

$$
\left.\left\langle\nabla_{T} S_{\theta}, S_{\theta}\right\rangle\right|_{\tau=\frac{\pi}{2}}=\left.\frac{1}{2} T\left\langle S_{\theta}, S_{\theta}\right\rangle\right|_{\tau=\frac{\pi}{2}}=0
$$

also by the proof of Lemma 6.3.

So, by the first derivative test, we see that $\left.\left\langle S_{\theta}, S_{\theta}\right\rangle\right|_{t=\tau}$ and $\left\langle\nabla_{T} S_{\theta}, S_{\theta}\right\rangle_{t=\tau}$ are of opposite sign, for all $\theta \in S^{2 n-1}$. (Keep in mind that $\left\langle S_{\theta}, S_{\theta}\right\rangle$ has no zeroes on $[0, \pi]$ by Lemma 6.3 if $\left\langle S_{\theta}, S_{\theta}\right\rangle \neq 0$ at $s=0, \tau=t$.) 
Thus, if $\left\langle S_{\theta}, S_{\theta}\right\rangle>0$ for $\tau=t, s=0$, then

$$
\begin{aligned}
0 & >\left\langle\nabla_{T}, S_{\theta}, S_{\theta}\right\rangle \quad \text { at } \tau=t, s=0 \\
& =\left\langle\nabla_{S_{\theta}} T, S_{\theta}\right\rangle=S_{\theta}\left\langle T, S_{\theta}\right\rangle-\left\langle T, \nabla_{S_{\theta}} S_{\theta}\right\rangle=S_{\theta}\left\langle T, S_{\theta}\right\rangle .
\end{aligned}
$$

So $\left\langle S_{\theta}, S_{\theta}\right\rangle>0$ at $\tau=t, s=0$ implies that $S_{\theta}\left\langle T, S_{\theta}\right\rangle<0$ at $\tau=t, s=0$ as required, and by the first part of this argument, $S_{\theta}\langle T, T\rangle<0$ for $\tau=$ $t, s=0$. Since $\langle T, T\rangle=0$ at $\tau=t, s=0$, we see that $\langle T, T\rangle<0$ for $\tau=t$ and for small $s>0$. (Actually, for $\tau=t,\left\langle T, S_{\theta}\right\rangle$ is a linear function of $S$, so $\langle T, T\rangle<0$ for $\tau=t$ and for all $s>0$ which correspond to points where the variation $V$ is defined.) Thus, the geodesic from $\gamma(0)$ to the point $V(\tau=t, s=0, \theta)$ is timelike if the one from $\gamma(t)$ is spacelike.

We must now show that the lightcone of $\hat{x} \in F$ projects onto a neighborhood of $x=\pi(\hat{x})$ in $M$. It will be convenient to deal with a suitable subset $\mathscr{L}$ of this lightcone, which we now construct.

Let $U$ be a normal neighborhood of $\hat{x}=\gamma(0)$ containing $\gamma\left(\left[-\frac{\pi}{2}-\varepsilon, \frac{\pi}{2}+\varepsilon\right]\right)$ for some $0<\varepsilon<\pi / 2$; the existence of $U$ is guaranteed by Lemma 7.2. Let $U_{1}$ be another such neighborhood whose closure is contained in $U$. Let $\Gamma$ be the vertical hypersurface consisting of all the translates $\Sigma_{t}$ of $\Sigma_{0} ; \Gamma=\pi^{-1}\left(\pi\left(\Sigma_{0}\right)\right)$. This $\Gamma$ divides $U_{1}$ into two disjoint open sets; let $\mathscr{L}^{+}$be the part of the lightcone of $\hat{x}$ contained in one of these sets, and let $\mathscr{L}^{-}$be the other. The set we will project will be the union $\mathscr{L}=\operatorname{cl}\left(\mathscr{L}^{+}\right) \cup \operatorname{cl}\left(\mathscr{L}^{-}\right)$of the closures of $\mathscr{L}^{+}$and $\mathscr{L}^{-} . \mathscr{L}$ is a closed subset of $F$, containing the intersection of the lightcone of $\hat{x}$ with the open neighborhood $U$.

In order to see more clearly the effect of the projection $\pi: F \rightarrow M$ on $\mathscr{L}$, it will be useful to know what happens when the lightcone $L C$ of the origin of Euclidean space $\mathbb{R}^{2 n+2}$ of signature $(2 p+1,2 q+1)$ is projected to $\mathbb{R}(2 n+1)$ along a family of parallel null lines. We will not consider the whole lightcone, but only the part contained in a closed ball of radius $\sqrt{2}$ about the origin. Let the vector field tangent to the family of parallel null lines be called $K_{1}$; we may arrange for $K_{1}$ to be a Killing field ("vertical vector field").

In this situation, the projection is a homeomorphism on $L C-K_{1}^{+}$. The null geodesics perpendicular to $K_{1}$ project to a cone on $S^{2 p-1} \times S^{2 q-1}$ in $\mathbb{R}^{2 n+1}$, and this image cone is tangent to the projection of $K_{1}^{+}$at the origin. We give explicit equations as follows:

$$
\begin{aligned}
L C & =\left\{a_{0}^{2}+\cdots+a_{2 p}^{2}-b_{0}^{2}-\cdots-b_{2 q}^{2}=0\right\}, \\
S^{2 n+1} & =\left\{a_{0}^{2}+\cdots+a_{2 p}^{2}+b_{0}^{2}+\cdots+b_{2 q}^{2}=2\right\} .
\end{aligned}
$$

We will find it easier to follow the calculation by projecting the boundary $B$ of $L C \cap S^{2 n+1}$ :

$$
B=\left\{a_{0}^{2}+\cdots+a_{2 p}^{2}=1, b_{0}^{2}+\cdots+b_{2 q}^{2}=1\right\} .
$$

$B$ is homeomorphic to $S^{2 p} \times S^{2 q}$. Let $t=a_{0}+b_{0}$ and let $s=a_{0}-b_{0}$. Then $a_{0}=\frac{1}{2}(t+s)$ and $b_{0}=\frac{1}{2}(t-s)$. The curves $s=a_{1}=\cdots=a_{2 p}=b_{1}=\cdots=b_{2 q}=$ constant ( $t$-coordinate curves) are null geodesics, and we choose these as the verticals; thus $K_{1}=\frac{\partial}{\partial t}$. Projecting to $\mathbb{R}^{2 n+1}=\{t=0\}$, we get

$$
\pi(B)=\left\{s= \pm \sqrt{1-a_{1}^{2}-\cdots-a_{2 p}^{2}} \pm \sqrt{1-b_{1}^{2}-\cdots-b_{2 q}^{2}}\right\}
$$

in $\mathbb{R}^{2 n+1}$ with coordinates $s, a_{1}, \ldots, a_{2 p}, b_{1}, \ldots, b_{2 q}$. This figure $\pi(B)$ can be seen to be homeomorphic to two copies of $S^{2 n}$ glued together along a truncated 
cone on $S^{2 p-1} \times S^{2 q-1}$ in the plane $s=0$. Filling in the interior of the two copies of $S^{2 n}$ gives us the projected image of $L C \cap S^{2 n+1}$.

We note that the projection we have just described does not map the lightcone of pseudo-Euclidean $\mathbb{R}^{2 n+2}$ of signature $(2 p+1),(2 q+1)$ onto a neighborhood of the origin in $\mathbb{R}^{2 n+1}$, since points of the plane $s=0$, other than those on the cone on $S^{2 p-1} \times S^{2 q-1}$ along with the two balls are glued, are not in the image. This, however, is not surprising, since Sparling's condition (2) $\operatorname{Ric}(K, K)>0$ is not satisfied for our choice of Killing field $K_{1}$ in pseudo-Euclidean space, and since as a consequence our Lemma 7.3 does not apply.

How does this picture differ in the Fefferman manifold $F$ of a nondegenerate CR hypersurface $M$ (of Levi signature $(p, q)$ ) in a neighborhood $U$ of $\hat{x}$ in $F$ ? The projection map $\pi: U \rightarrow M$, restricted to the lightcone $\mathscr{L}$ of $\hat{x}$, is still a local homeomorphism, except on $\mathscr{L} \cap \Gamma$. In fact, we claim that $\pi$ restricted to $\mathscr{L}^{+}$or $\mathscr{L}^{-}$is a homeomorphism.

Lemma 7.4. $\pi$ restricted to $\mathscr{L}^{+}$or $\mathscr{L}^{-}$is a homeomorphism.

$\operatorname{Proof}\left(\right.$ for $\left.\mathscr{L}^{+}\right)$. Let $\phi(t)$ be a fiber of $F$, with $\phi([a, b]) \subset U_{\hat{x}}$. We must show that there cannot exist two null geodesics $\alpha(s), \beta(s)$ with $\alpha(0)=\beta(0)=\hat{x}$, $\alpha(1)=\beta(1)=\phi(b)$, and $\langle K, \alpha\rangle>0,\langle K, \beta\rangle>0$.

So let us assume on the contrary that such geodesics $\alpha$ and $\beta$ do exist. Since $\phi([a, b]) \subset U_{\hat{x}}$, we may construct a variation $V(s, t)$ of geodesics from $\hat{x}$ to $\phi(t)$ for $s \in(0,1)$ and $t \in[a, b]$, with $V(s, a)=\alpha(s), V(s, b)=\beta(s)$, $V(0, t)=\hat{x}$, and $V(1, t)=\phi(t)$. Let $S, T$ be the tangents to the $s$ and $t$ curves, respectively. Then $\langle S, S\rangle=0$ for $t=a$ and $t=b$, since $\alpha$ and $\beta$ are null.

Now $T\langle S, S\rangle=2 S\langle T, S\rangle \not \equiv 0$ on $\phi([a, b])$, for if it were, then we would have $\langle T, S\rangle \equiv 0$ along $\phi$ (since $T=0$ if $s=0$ ). But we have assumed $\langle T, S\rangle>0$ for $t=a$ and $t=b$, for all $s$. Without loss of generality, we may assume that $\langle S, S\rangle$ has no zeroes for $t \in(a, b)$.

By the mean value theorem there is a $t_{0} \in(a, b)$ such that $T\langle S, S\rangle=0$ at $t=t_{0}$, for all $s$. Along the geodesic $V\left(s, t_{0}\right)$, we have $\langle T, S\rangle \equiv 0$, since $T=0$ at $s=0$, and $T\langle S, S\rangle=2 S\langle T, S\rangle \equiv 0$.

Now $T T\langle T, S\rangle \equiv 0$ along $\phi$ (by Jacobi's equation), so $T\langle T, S\rangle$ is constant on $\phi$. That is, $\langle T, S\rangle$ is a linear function of $t$ along $\phi$. But we have assumed that $\langle T, S\rangle>0$ for $t=a$ and $t=b$, and we have shown that there is a $t_{0} \in(a, b)$ so that $\langle T, S\rangle=0$ at $t=t_{0}$. This is impossible; therefore there cannot exist two null geodesics $\alpha$ and $\beta$ in $\mathscr{L}^{+}$with $\pi(\alpha(1))=\pi(\beta(1))$. Thus $\pi$ is a homeomorphism on $\mathscr{L}^{+}$.

We now see that $\pi\left(\mathscr{L}^{+}\right)$and $\pi\left(\mathscr{L}^{-}\right)$are the disjoint homeomorphic images of two $(2 n+1)$-balls in $M$. And, despite the fact that a one-parameter family of null geodesics perpendicular to $K$, whose initial tangent vectors have collinear projections, no longer project to the same curve, nevertheless Lemma 6.1 assures us that the set of all perpendicular null geodesics through $\hat{x}$ in $U$ projects to a (truncated) cone on $S^{2 p-1} \times S^{2 q-1}$ in $\pi(\Sigma)$. (Here $\Sigma$ is analogous to the surface $s=0$ in the hyperquadric.)

The projection $\pi: F \rightarrow M$ restricted to $\mathscr{L}$ fails to behave identically to the map described on pseudo-Euclidean space exactly because of Lemma 7.3. Lemma 7.3 tells us that all fibers through regions (1) and (2) of $\Sigma$ sufficiently near to $\gamma=\pi^{-1}(x)$ intersect both $\operatorname{cl}\left(\mathscr{L}^{+}\right)$and $\operatorname{cl}\left(\mathscr{L}^{-}\right)$. Being in $\Gamma$, these 
points of intersection are on the boundaries of $\operatorname{cl}\left(\mathscr{L}^{+}\right)$and $\operatorname{cl}\left(\mathscr{L}^{-}\right)$. As we have noted above, all fibers through region (3) near $\gamma$ are on the boundaries of $\operatorname{cl}\left(\mathscr{L}^{+}\right)$and $\operatorname{cl}\left(\mathscr{L}^{-}\right)$where they lie in $\operatorname{cl}\left(U_{1}\right)$. Thus we see that a neighborhood of $x=\pi(\gamma)$ in $\pi(\Sigma)$ is contained in the intersection of the boundaries of $\pi\left(\operatorname{cl}\left(\mathscr{L}^{+}\right)\right)$and $\pi\left(\operatorname{cl}\left(\mathscr{L}^{-}\right)\right)$. Aside from these points, $\pi\left(\operatorname{cl}\left(\mathscr{L}^{+}\right)\right)$and $\pi\left(\operatorname{cl}\left(\mathscr{L}^{-}\right)\right)$are disjoint. So we see that $\pi$ maps $\mathscr{L}^{+} \cup \mathscr{L}^{-}$to a figure homeomorphic to two closed $(2 n+1)$-balls in $M$, glued together along a $(2 n)$-disc on their boundaries, with $x=\pi(\hat{x})$ in the interior. Since all points of this ball can be reached from $x$ by the projection of a null geodesic of $F$, we see that $x$ can be joined to every point in the ball by either a chain or a null-chain.

\section{REMARKS}

In conclusion, we would like to make two remarks. First, the results on the nature of null-chains and the two connectivity theorems contained in this work also hold for the more general systems of curves called pseudochains (and pseudo-null-chains) defined on nondegenerate CR manifolds in [Ko]. These non-CR-invariant systems of curves on a CR manifold $M$ are obtained by projecting null geodesics of pseudo-Riemannian metrics $\mathrm{g}$ on a circle bundle over $M$ which satisfy the following conditions:

$\left(0^{\prime}\right)$ The vertical vector field $K$ is Killing: $\mathscr{L}_{K} \mathrm{~g}=0$.

(1') $\mathrm{g}(\operatorname{Weyl}(K, X) X, K)=0$ for all $x$ with $\mathrm{g}(K, X)=0$.

(2') $\operatorname{Ric}(K, K)>0$.

These conditions are nearly identical to Sparling's conditions, but $\left(1^{\prime}\right)$ is considerably weaker than Sparling's condition (1), so metrics $g$ may satisfy these conditions without being Fefferman for $M$. Nevertheless, only these three conditions are required to define pseudochains (and pseudo-null-chains) on $M$, and to show that they behave like chains (and null-chains) in the ways described in this paper.

Second, we might ask what the behavior of the null-chains on an indefinite CR manifold $M$ can tell us about the possible existence of holomorphic submanifolds of $M$. It is well known that, if $M$ contains a holomorphic submanifold $N$ passing through a point $x \in M$, then the tangent space $N_{x}$ to $N$ at $x$ consists of null vectors of the Levi form of $M$ at $x$; i.e., $\left.\mathrm{L}\right|_{N_{x}}=0$. Let $V \in N_{x}$; then, since $\mathrm{L}(V, V)=0$, we know that a one-parameter family of null-chains of $M$ pass through $x$ tangent to $V$. (Of course, null chains exist even if $M$ admits no holomorphic submanifolds.)

Do the null-chains tangent to $V$ at $x$ remain in $N$ if $V \in N_{x}$ ? Equivalently, is the totally null submanifold $\tilde{N}$ of $F$ defined by $\tilde{N}=\pi^{-1}(N)$ a totally geodesic submanifold? (Here $F$ is the Fefferman manifold of $M$.) The answer is (1) yes if $M$ is of "split" Levi signature $(p, p)$ and $N$ has complex dimension $p$, but (2) no in general. For in case (1), $N$ lifts to a totally null submanifold of real dimension $2 p+1$ in $F$, the signature of $F$ is $(2 p+1,2 p+1)$, and it is known (see e.g., $[\mathrm{Ku}]$ ) that a $k$-dimensional totally null submanifold of a pseudo-Riemannian manifold of signature $(k, k)$ is necessarily totally geodesic. On the other hand (2), this cannot hold for $M$ of general signature and $N$ of any dimension, since it fails for holomorphic curves in $M$ of dimension $(2 n+1), n \geq 3$, and signature $(1, n-1)$. R. Bryant shows in 
[B] that CR manifolds of this signature (which he calls Lorentzian) admit (at most) an $n^{2}$-parameter family of holomorphic curves, and he gives examples where this maximum is achieved. Since the Fefferman manifold $F$ of an $M$ of this dimension and signature admits (at most) a $(4 n-4)$-parameter family of totally null, totally geodesic vertical submanifolds of dimension 3 (this maximum is achieved for the Fefferman manifold of the hyperquadric $\left.Q_{1, n-1}\right)$, we see that $M$ may admit too many holomorphic curves for our conjecture to hold in general. However, it remains possible that null-chains and holomorphic submanifolds are related in some more complicated way.

\section{REFERENCES}

[B] R. Bryant, Holomorphic curves in Lorentzian CR-manifolds, Trans. Amer. Math. Soc. 272 (1982), 203.

[BDS] D. Burns, K. Diederich, and S. Shnider, Distinguished curves in pseudoconvex boundaries, Duke Math. J. 43 (1977), 407-431.

[BS] D. Burns and S. Shnider, Real hypersurfaces in complex manifolds, Several Complex Variables, Proc. Sympos. Pure Math., Vol. 40, Part 2, Amer. Math. Soc., Providence, R.I., 1977, p. 141.

[Ca] É. Cartan, Sur la géométrie pseudo-conforme des hypersurfaces de deux variables complexes. I, Euvres Complètes, Gauthier-Villars, Paris, 1955, pp. 1231-1304; II, Euvres Complètes, Gauthier-Villars, Paris, 1955, pp. 1217-1238.

[Ch] J.-H. Cheng, Chain-preserving differomorphisms and CR equivalence, preprint.

[CM] S. S. Chern and J. K. Moser, Real hypersurfaces in complex manifolds, Acta Math. 133 (1974), 219-271.

[Fa] F. Farris, An intrinsic construction of Fefferman's CR metric, Pacific J. Math. 123 (1986), 33.

[Fe] C. Fefferman, Monge-Amperre equations, the Berman kernel, and the geometry of pseudoconvex domains, Ann. of Math. 103 (1976), 395; Erratum, 104 (1976), 393.

[G] C. R. Graham, On Sparling's characterization of Fefferman metrics, Amer. J. Math. 109 (1897), 853-841.

[H] S. Harris, A triangle comparison theorem for Lorentz manifolds, Indiana Univ. Math. J. 31 (1982), 289.

[J] H. Jacobowitz, Chains in CR geometry, J. Differential Geometry 21 (1985), 163.

[Ko] L. Koch, Chains in CR geometry and Lorentz manifolds, Trans. Amer. Math. Soc. 307 (1988), 827.

[Kr] N. Kruzhilin, Local automorphisms and mappings of smooth strictly pseudoconvex hypersurfaces, Math. USSR-Izv. 26 (1986), 531.

[Ku] D. Kupeli, Degenerate submanifolds in semi-Riemannian geometry, Geom. Dedicata 24 (1986), 337.

[L] J. Lee, The Fefferman metric and pseudohermetian invariants, Trans. Amer. Math. Soc. 296 (1986), 411.

[P] H. Poincaré, Les fonctions analytiques de deux variables et la représentation conforme, Rend. Circ. Mat. Palermo (1907), 185.

[S] G. Sparling, Twistor theory and the characterization of Fefferman's conformal structures, preprint.

[W] S. Webster, Pseudo-Hermitian structures on a real hypersurface, J. Differential Geometry 13 (1978), 25.

Department of Mathematics and Statistics, University of Pittsburgh, Pittsburgh, PenNSYlVania 15260

E-mail address: lkoch+@pitt.edu 